\title{
Les « libros de textos » de l'Espagne du XXe sicècle et la recherche historique
}

Sylvie Durando

\section{OpenEdition}

1 Journals

Édition électronique

URL : http://journals.openedition.org/trema/1824

DOI : $10.4000 /$ trema. 1824

ISSN : 2107-0997

Éditeur

Faculté d'Éducation de l'université de Montpellier

\section{Édition imprimée}

Date de publication : 1 décembre 1998

Pagination : 91-98

ISSN : 1167-315X

\section{Référence électronique}

Sylvie Durando, "Les « libros de textos » de l'Espagne du XXe sicècle et la recherche historique »,

Tréma [En ligne], 14 | 1998, mis en ligne le 01 décembre 1998, consulté le 20 avril 2019. URL : http:// journals.openedition.org/trema/1824 ; DOI : 10.4000/trema.1824

Ce document a été généré automatiquement le 20 avril 2019

Trema 


\title{
Les « libros de textos » de l'Espagne du $\mathrm{XXe}$ sicècle et la recherche historique
}

\author{
Sylvie Durando
}

1 Les travaux sur l'histoire de l'enseignement sont fondamentaux pour la connaissance des sociétés $\mathrm{du} \mathrm{XIX}^{\mathrm{e}}$ et, surtout $\mathrm{du} \mathrm{XX}^{\mathrm{e}}$ siècle : les très nombreuses études que les historiens ont menées depuis longtemps sur ce sujet en sont la preuve.

\section{Enjeux et contexte}

2 Pour aborder ce champ de l'histoire, les manuels scolaires - notamment ceux utilisés au cours de la scolarité obligatoire - représentent une source de choix. On doit prendre en compte le fait qu'ils constituent le socle commun de références de toute une génération, ce qui n'est pas le cas des autres productions culturelles de masse dont la diffusion n'est pas aussi générale. L'antériorité de leur réception n'est pas non plus négligeable: les traces laissées dans les jeunes esprits par les manuels scolaires sont d'autant plus profondes qu'elles sont précoces. D'autre part, on peut estimer à juste titre que ces ouvrages présentent un reflet assez fidèle des discours que les maîtres tiennent aux enfants. En ce sens, ils sont plus proches de la réalité pédagogique telle qu'elle fut vécue au quotidien dans les classes que les instructions des programmes officiels qui sont des documents plus politiques parce qu'ils ont été conçus par le Ministère de l'Education et donc par le gouvernement.

D'un point de vue pratique enfin, ils constituent une source assez stable et abondante: pour le $\mathrm{XX}^{\mathrm{e}}$ siècle, on peut assez facilement reconstituer des collections et des séries continues qui couvrent les périodes que l'on souhaite étudier.

4 Tous ces avantages expliquent sans doute que les études menées à partir des manuels scolaires et recherchant quels sont les savoirs et les valeurs qu'une société cherche à inculquer et à transmettre aux enfants sont assez nombreuses et anciennes, en particulier en France ${ }^{1}$. A quoi il convient d'ajouter d'autres facteurs, moins scientifiques, notamment l'attendrissement rétrospectif éprouvé par nos concitoyens à l'égard des anciens 
systèmes scolaires, un sentiment très répandu en France dont témoignent l'extraordinaire succès de librairie des pittoresques anthologies que Gaston Bonheur a consacrées à l'école de la III ${ }^{\mathrm{e}}$ et de la IV ${ }^{\mathrm{e}}$ République - Qui a cassé le vase de Soisson ? et La République nous appelle ${ }^{2}$ - et les innombrables rééditions à l'identique d'un manuel comme Le Tour de la France par deux enfants.

Figure 1 : Une approche subjective et passionnée de l'univers des « libros de textos »de l'époque franquiste.

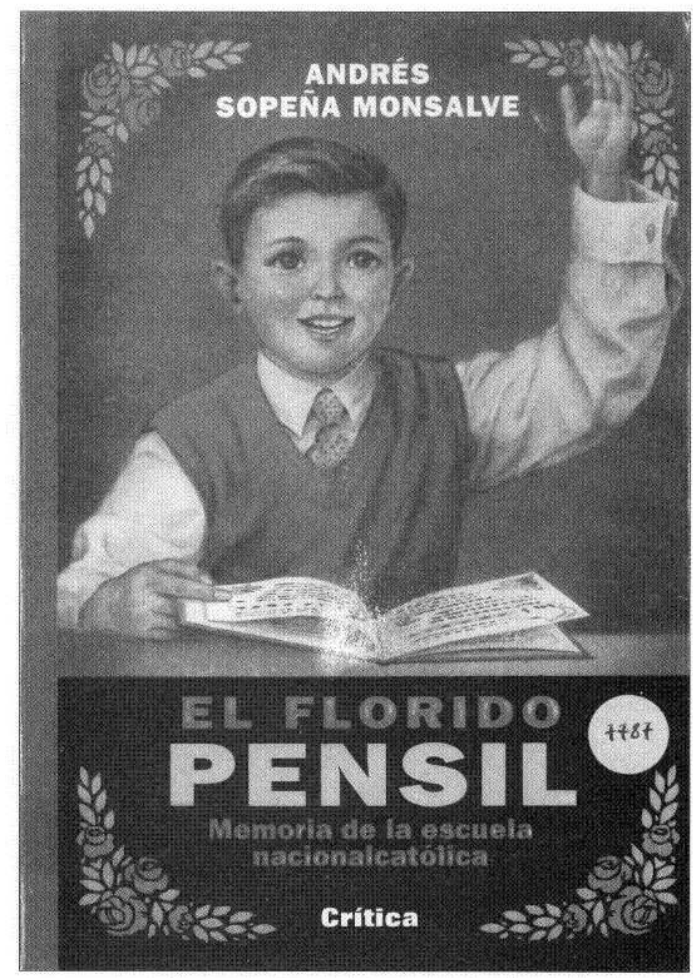

SOPEÑA MONSALVE A. : El Florido pensil.Barcelona, Grupo Grijalbo-Mondadori, collección Crítica, 1994, 243 p., première de couverture.

5 On trouve aujourd'hui, en Espagne, un engouement comparable pour le passé scolaire national. Ainsi Andrés Sopeña Monsalve a proposé un regard mi-critique mi-nostalgique sur l'école au temps du franquisme à partir d'une analyse de ses manuels ${ }^{3}$; et l'on vient de rééditer en fac simile, avec un certain succès, un classique de la production pédagogique, l'Enciclopedia escolar intuitiva, sintética y práctica. d'Antonio Alvarez Pérez ${ }^{4}$. Le meilleur indice de cette curiosité est le fait que, sur le marché des livres anciens, la cote des manuels scolaires est beaucoup plus élevée en Espagne qu'en France...

6 Toutefois les recherches universitaires sur ce terrain n'ont commencé en Espagne que beaucoup plus tard, notamment à cause de la censure franquiste - ce qui prouve à quel point ces questions sont idéologiquement «sensibles» - et elles se sont d'abord intéressées aux manuels destinés à l'enseignement de la littérature ${ }^{5}$ ou de la morale. Ajoutons que, quantitativement, ces études sont encore relativement peu nombreuses.

7 Pourtant, les circonstances politiques générales et les conditions particulières de l'enseignement au cours du $\mathrm{XX}^{\mathrm{e}}$ siècle en Espagne font que de telles investigations s'avéreraient particulièrement utiles et fructueuses. 
En effet, le pouvoir espagnol a toujours été très attentif à l'enseignement national et à ses contenus. Les « libros de textos », ainsi qu'on appelle les ouvrages scolaires espagnols, ont toujours été contrôlés. Alors que l'usage des manuels scolaires avait été rendu obligatoire dès 1845 , un contrôle précis de la production pédagogique par le Ministère de l'Education a été institué en $1899^{6}$. Et cette surveillance est devenue encore plus rigoureuse et systématique lors de la dictature de Primo de Rivera, puis sous celle du général Franco où la critique du système éducatif dans son ensemble prend un tour particulièrement dramatique : on purge le corps enseignant de ses éléments indésirables, on vérifie la composition des bibliothèques scolaires, on détruit les ouvrages " dangereux $»^{7}$ et ceux écrits dans une autre langue que le castillan, et on inspecte systématiquement les écoles pour vérifier l'exécution pointilleuse de ces ordres... ${ }^{8}$ Autant de mesures drastiques, qui ont contribué à fixer de manière très stricte les contenus des manuels scolaires. Cela est particulièrement vrai pour les manuels d'histoire, à tel point que l'historien Manuel Tiñón de Lara a pu, à juste titre, qualifier l'histoire enseignée à cette époque de «sierva de la enseñanza política y eclesiástica »[esclave de l'enseignement politique et ecclésiastique].

Figure 2 : Le Christ et le Caudillo surveillent l'univers scolaire espagnol...

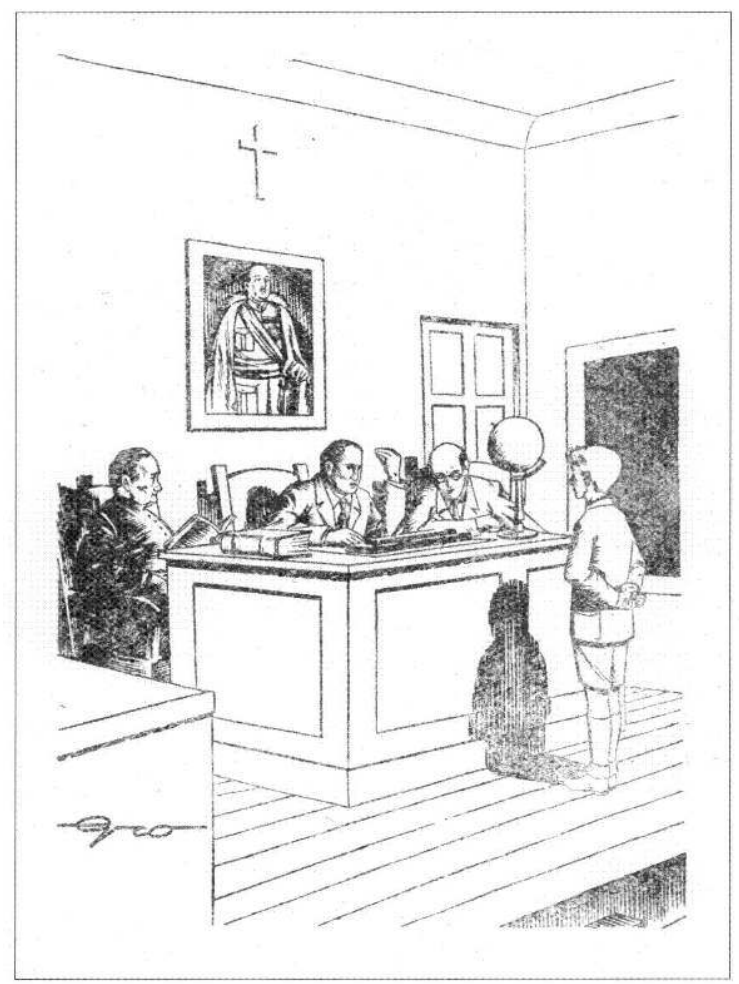

BARBERÁN CASTRILLO M. : Rayos de Luz. Madrid, Juan Bravo, 1951, 209 p., p. 201.

\section{La tyrannie des manuels}

Dans ces conditions, le manuel scolaire exerce une véritable tyrannie, dans la mesure où les discours proposés par les manuels étaient certainement peu modifié par les enseignants. D'une part ceux-ci étaient très étroitement surveillés et, d'autre part, étant donnée la médiocrité de leur formation initiale, leurs connaissances théoriques étaient très limitées ${ }^{10}$. L'instruction des enseignants, surtout au niveau du premier degré, était 
notoirement rapide, dogmatique et sommaire, si bien que les ouvrages scolaires à l'usage des élèves, éventuellement complétés par des « livres du maître » dans les établissements les mieux dotés, étaient leur unique point de repère lorsqu'ils préparaient leurs leçons ${ }^{11}$. Dans ces conditions, la pédagogie élémentaire était surtout basée sur la mémorisation fidèle et la restitution du texte du manuel.

Enfin, à une époque où la formation scolaire était assez courte, le «libro de texto » était destiné à être conservé par le futur adulte pour l'accompagner sa vie durant: il constituait ainsi une sorte de somme du savoir élémentaire. Cet usage postscolaire était particulièrement net pour les «enciclopedias escolares » - l'équivalent espagnol des « livres uniques » français - qui regroupaient, en un seul volume, tout sur toutes les matières, de l'arithmétique à la langue castillane en passant par les sciences naturelles et la religion... Ces ouvrages particuliers avaient été conçus au départ pour que les parents n'aient pas à acheter trop de livres à leurs enfants et leur usage fut assez répandu puisque une loi de 1972 réaffirma le bien fondé de ce principe pédagogico-économique, au grand dam de certains éditeurs scolaires ${ }^{12}$. Nul doute que leurs illustrations, souvent abondantes, toujours naïves, à force d'être vues et revues, acquéraient une force singulière ${ }^{13}$...

11 Sans doute, dans le contexte économique souvent difficile de l'Espagne de la première moitié du XXe siècle, on peut douter que tous les enfants scolarisés aient disposé à toutes les époques de "libros de textos»" Il n'en reste pas moins que l'examen de cette production est capitale pour la compréhension des mentalités en général. Les manuels d'Histoire en particulier, qui sont souvent les vecteurs d'une instruction civique avouée ou latente, visent, en exaltant un passé commun, à assurer la cohésion sociale du présent mais aussi et surtout à forger une communauté unie pour le futur. Dans la perspective de la "Transition démocratique", qui a mobilisé les intellectuels espagnols pour une exploration critique de la mémoire du franquisme, l'examen de cette production prend des allures de devoir civique.

\section{Les voies de la recherche historique}

D'une manière générale, les manuels d'histoire peuvent être étudiés de deux point de vue, pédagogique ou mythologique.

Ainsi, dans un champ disciplinaire donné, les didacticiens peuvent adopter un point de vue historique tout en considérant les ouvrages scolaires pour ce qu'ils sont: des outils d'enseignement. Dans cette perspective fonctionnelle, ils analysent la production scolaire en tant que pédagogues. Leurs mobiles sont d'ordre épistémologique et moral.

D'une part, en effet, ils ont conscience que, comme toute construction du savoir, la définition d'une théorie et d'une pratique enseignante se fait polémiquement, à partir d'une critique des principe et des usages établis, et que l'on se condamne, dès lors qu'on se cantonne à l'étude du présent, à ne pas comprendre les raisons des changements intervenus.

D'autre part, mesurant que toute doctrine didactique est précaire parce qu'elle s'inscrit dans une histoire, ils ont à cœur de ménager l'avenir professionnel des enseignants : en ménageant une perspective historique, ils permettent à l'instituteur ou au professeur d'aujourd'hui un retour critique sur les méthodes et les techniques de classe pratiquées qui ne prendra pas la forme d'une révision déchirante et ne débouchera pas sur une culpabilisation malsaine. Observons en passant qu'il est regrettable que la formation 
professionnelle initiale des enseignants dans les I.U.F.M., aveuglée par l'urgence des difficultés présentes, ne ménage pas de telles perspectives qui garantiraient la formation continue de demain.

Cependant, le manuel scolaire peut aussi être envisagé d'un point de vue plus idéologique comme un matériau historique étudié diachro-niquement ou synchroniquement.

L'approche synchronique est sans doute la plus courante et la plus ancienne. Ainsi Claude Billard, Pierre Guibbert ${ }^{15}$ et Christian Amalvi ${ }^{16}$, dressant la galerie des héros de l'Histoire de France sous la III et sous la $\mathrm{V}^{\mathrm{e}}$ République, ont-ils élucidé les présupposés de leur présentation. Jacqueline Freyssinet-Dominjon, pour sa part, a comparé minutieusement des séries de manuels d'Histoire laïques et confessionnels ${ }^{17}$. Quant à Marc Ferro ${ }^{18}$, il a confronté des ouvrages de différents pays pour déterminer les spécificités nationales de l'enseignement de l'histoire. L'inconvénient majeur de ce type de démarche, qui met à profit la stabilité du discours scolaire, est qu'elle finit par brosser des tableaux presque intemporels. En d'autres termes, on est davantage ici sur le terrain des mythologies que dans le champ de l'histoire.

On observe heureusement, depuis quelques années, une tendance de la recherche à prendre en compte l'évolution des contenus, à définir des lignes de fracture susceptibles de servir de cadre à une périodisation spécifique, bref, à jeter les bases d'une histoire des didactiques originale.

\section{Sources et méthode}

Ces préoccupations supposent l'existence d'archives riches, disponibles et systématiquement organisées. $\mathrm{Si}$, comme nous l'avons vu, les matériaux scolaires sont encore peu exploités en Espagne, alors que les obstacles politiques sont aujourd'hui levés, c'est parce qu'il n'y a pas dans ce pays, comme en France, des institutions qui regroupent des collections anciennes de manuels ${ }^{19}$ de sorte que les chercheurs qui souhaitent s'engager dans cette voie sont contraints de travailler à partir de collections privées ou des fonds de la Biblioteca National de Madrid,mais cette archive ne collectionne pas systématiquement ce genre d'ouvrages. 
Figure 3

Un poncif de la morale franquiste rendu intelligible aux enfants de l'école primaire : l'or est de peu de poids au regard de la dignité humaine...

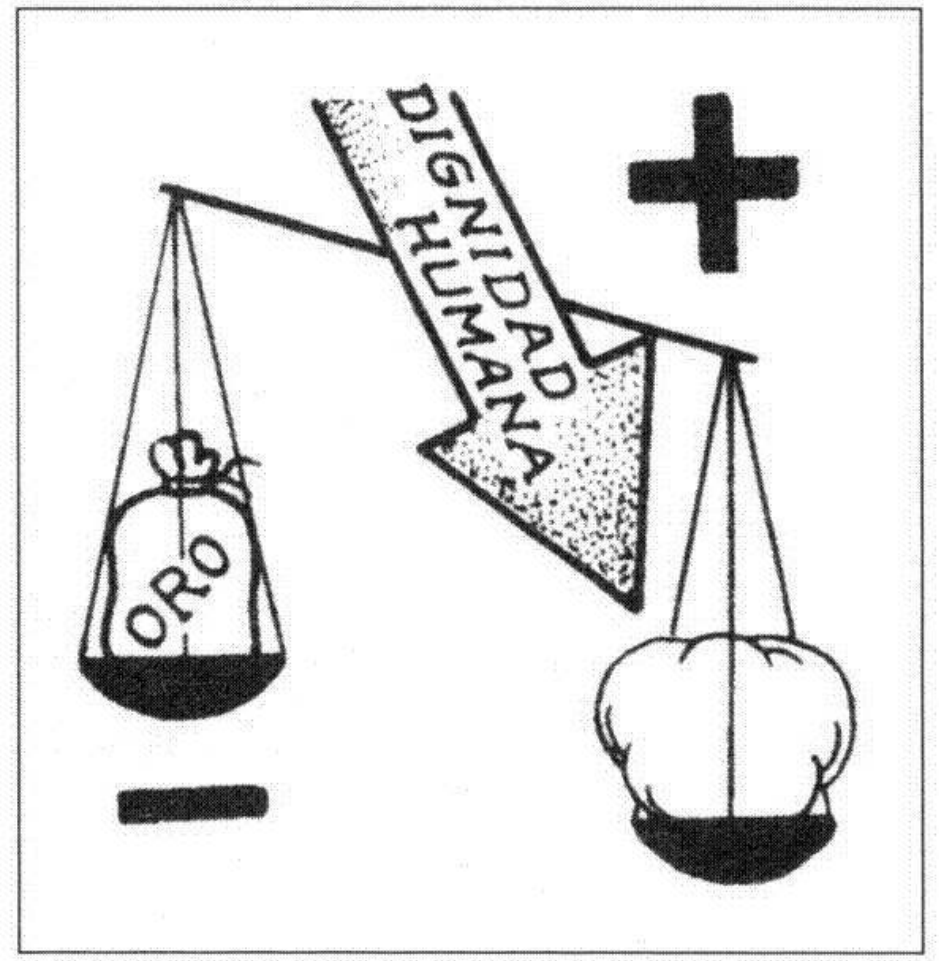

ALVAREZ PÉREZ A. :Enciclopedia escolar intuitiva, sintética y práctica - Tercer grado. Valladolid, Mirón s.a., 1966,635 p., p. 596

\section{l'évaluation de la représentativité d'un corpus cohérent de "libros de textos ${ }^{20}$. Le. historiens espagnols ont hésité à dresser des listes d'ouvrages de référence, sans doute} parce qu'une telle entreprise pose des problèmes théoriques et pratiques difficiles à résoudre.

Idéalement, il conviendrait de retrouver toutes les listes de manuels proprement dits «libros de textos» - et d'ouvrages "declarados de textos» - c'est-à-dire des ouvrages non scolaires autorisés par les différents Ministres de l'Education et utilisés dans les écoles pour pallier la pénurie de manuels. Mais on n'aboutirait ainsi - pour autant qu'on retrouverait ces listes - qu'à dresser une bibliographie virtuelle. Cependant, l'établissement d'une bibliographie réelle, par la collation des données relevées dans les manuels effectivement rassemblés, soulève bien des problèmes. Ainsi, la détermination de l'origine éditoriale est souvent impossible : dans nombre de cas, seule la raison sociale de l'imprimeur ou d'un distributeur - en général un libraire - est fournie. D'autre part les indications de niveau, surtout pour les périodes les plus reculées, font souvent défaut ou ne sont pas fiables. Enfin on ne sait pas toujours à quoi correspondent les dates mentionnées sur les ouvrages anciens : s'agit-il de la date de la première édition? de la date d'une édition postérieure largement décalée dans le temps? Ajoutons qu'à l'occasion d'une réédition certains ouvrages ont été "revus et corrigés", parfois par d'autres personnes que l'auteur, sans que ces transformations soient signalées par l'éditeur. 
21 A vrai dire l'absence de ces indications n'est pas très dommageable dès lors qu'on se place du point de vue de la réception des textes et non du point de vue de leur élaboration. En effet, il convient de prendre en compte que les manuels scolaires, surtout à la « haute époque» de la scolarisation de l'Espagne, demeurent souvent en circulation très longtemps après leur date de parution, et parfois même très longtemps après les dates de modification des programmes, pour d'évidents motifs économiques et aussi en raison de l'immobilisme pédagogique de certains maîtres. Ceci reste vrai pour les périodes récentes, malgré des dispositions légales qui prévoyaient une durée de validité de quatre ans maximum pour les « libros de textos ».

Ces difficultés expliquent que les travaux historiques sur la production scolaire espagnole sont pour le moment peu capables d'enregistrer des variations fines sur de brèves périodes - le temps de la pédagogie est un temps relativement long - et que les perspectives offertes soient plus idéologiques que proprement historiques.

\section{NOTES}

1. Je donnerai plus loin quelques exemples de ces travaux. Pour disposer d'une base comparative on peut consulter : SELANDER S. : " Análisis de textos pedagógicos, hacia un nuevo enfoque de la investigación educativa ", in Revista de la Educación, № 293, septiembre-diciembre 1990, pp. 345-354, qui donne une bibliographie critique des travaux portant sur les productions suédoises et anglo-saxonnes. Pour des exemples de recherche conduites dans cette perspective, $C f$ : DURANDO S. et GUIBBERT P.: «Entrer dans l'Histoire. Les couvertures des manuels scolaires espagnols et français (1880-1980)», in "Nationale Identitäten", Internationale Schulbuchforschung, $\mathrm{N}^{\circ}$ 3, Vol. 18. Frankfurt, Diesterweg, 1996, pp. 387-410; DURANDO S. et GUIBBERT P. : «Les sommaires d'une histoire sommaire : les tables des matières des manuels de la première Histoire (Allemagne, Angleterre, Espagne, France, Italie)», in Internationale Schulbuchforschung, à paraître en mars 1999.

2. BONHEUR G.: Qui a cassé le vase de Soissons ? Paris, Robert Laffont, 1963, 447 p.; et La République nous appelle. Paris, Robert Laffont, 1965, 383 p.

3. SOPEÑA MONSALVE A. : El Florido pensil, memoria de la escuela nacionalcatólica. Barcelona, Grupo Grijalbo-Mondadori, collección Crítica, 1994, 243 p.

4. ALVAREZ PÉREZ A. : Enciclopedía escolar intuitiva, sintética y práctica - Tercer grado, Edition originale. Valladolid, Mirón s.a., 1966, 635 p. Reprint à l'identique : Madrid, EDAF, 1997, 635 p.

5. Je pense notamment aux travaux de : VALLS F. : La enseñanza de la literatura en el franquismo 1936-1951. Barcelona, Antoni Bosch, 1983, 198 p. ; et de GARCÍA CRESPO C. : Léxico e ideología en los libros de lectura en la escuela primaria 1940-1975. Salamanca, Universidad de Salamanca, 1983, $183 \mathrm{p}$.

6. Cf., GONZALEZ I. et ZARAGOZA G. : Cuadernos de Pedagogía, № 122, febrero 1985, pp. 4-6.

7. « < La depuración de las bibliotecas escolares se dispone ya en la orden ministerial del 4 de septiembre de 1936. A la luz des las instrucctiones cursadas, sólo deberán subsistir aquellos libros que respondan a los principios de la religión y a la moral cristiana y exalten el patriotismo `. Una vez más en nuestra historia, se reproducía [...] la vieja división maniquea de libros buenos y malos". Traduction : «〈La purge des bibliothèques scolaires est déjà arrêtée par la circulaire 
ministérielle du 2 septembre 1936. Conformément aux indications promulguées, seuls devront subsister les livres qui correspondent aux principes de la religion et à la morale chrétienne, et exaltent le patriotismes. Une fois de plus, dans notre histoire, on reproduisait [...] l'antique distinction entre les bons et les mauvais livres. " DE PUELLES BENITEZ M. : Educación e idéología en la España contemporánea. Barcelona, Ed. Labor, 1986, 468 p., p. 367.

8. CAMARA VILLAR G. : Nacional catolicismo y escuela. Jaen, Hesperia, 1984 ; et ALTED VIGIL A. : Política del nuevo estado sobre el patrimonio cultural y la educación durante la guerra civil española.Madrid, Ministerio de la Cultura, 1984, 391 p. - détaillent ces mesures.

9. TUÑON DE LARA M. : «La interpretación policial de la historia », in Cuadernos de Pedagogía, Numéro spécial « Educación y fascismo », pp. 35-37.

10. Les livres de PÉREZ GALÁN M. : La Enseñanza en la Segunda república. Madrid, Mondadori, 1977, 335 p. ; et de LOZANO C. : Escuela republicana. Barcelona, Universidad de Barcelona, 1980, 456 p. - analysent le système éducatif de ces années-là.

11. Cf., PALACIOS BAÑUELOS L. : «El mundo de los valores en la Institución Libre de Enseñanza », in Boletín de la Real Academia de Córdoba de Ciencias, Bellas Artes y Nobles Artes. $\mathrm{N}^{\circ} 116$, enerojunio 1989, pp. 37-49.

12. Cf., LUZÁN J.: "Libros de textos, batalla entre editores y libreros ", in Cuadernos de Pedagogía, $\mathrm{N}^{\circ} 23$, febrero 1976, pp. 34-35.

13. Des formules adaptées à l'enseignement des adultes ont existé. Il y eut ainsi des encyclopédies spécialement consacrées aux femmes de la Phalange qui regroupaient, à côté de notions de religion, d'Histoire de l'Espagne et de politique, des leçons d'hygiène, d'économie ménagère et de cuisine.

14. Une enquête réalisée par le "Collectiu d'Educatio" - La enseñanza en España, Madrid, Alberto Corazón, 1975, 372 p., - dresse un inventaire de ces problèmes.

15. BILLARD C, GUIBBERT P.: L'Histoire mythologique des Français. Paris, Galilée, collection Coups pour coups, 1976, $309 \mathrm{p}$.

16. AMALVI C. : Les héros de l'Histoire de France. Paris, Phot'Æil, 1979, 315 p.; et De l'art et la manière d'accommoder les héros de l'Histoire de France. De Vercingétorix à la Révolution. Essais de mythologie nationale. Paris, Albin Michel, 1988, $473 \mathrm{p}$.

17. FREYSSINET-DOMINJON J. : Les manuels d'Histoire de l'école libre 1882-1959. Paris, Armand Colin, Fondation Nationale des Sciences politiques, 1969, $294 \mathrm{p}$.

18. FERRO M. : Comment on raconte l'Histoire aux enfants du monde entier. Paris, Payot, 1981, 316 p.

19. Il a existé en Espagne un "Museo pedagógico Nacional », dirigé au départ par Manuel B. Cossio. Cette institution, qui avait été créée pour promouvoir la recherche pédagogique, a été fermée et ses collections arrêtées. La Revista de Educación ( $\left.\mathrm{N}^{\circ} 288-294\right)$ a publié un inventaire de ses fonds. L'université de Salamanca, qui dispose d'une importante revue spécialisée, Historia de la Educación, avait formé le projet de fonder un Centre de Recherches en histoire de l'Enseignement, mais, à ma connaissance, elle ne dispose pas d'une base documentaire spécialisée.

20. Buenaventura DELGADO, dans son article « Los libros de textos como fuente para la Historia de la Educación ", in Historia de la Educación, Nº 2 (Salamanca, 1983, pp. 353-358), insiste sur la nécessité de mener à bien un tel travail. 


\section{RÉSUMÉS}

Non disponible

Not available

INDEX

Keywords : history, hitorical research, school textbook, Spain

Mots-clés : Espagne, histoire, manuel scolaire, recherche historique

\section{AUTEUR}

SYLVIE DURANDO

Université de Perpignan, équipe DIDAXIS, Montpellier 